\title{
INDIVIDUAL SUPPORT FOR INDEPENDENT LEARNING AT THE LANGUAGE CENTER AT UNIL: FROM TEACHING TO ADVISING
}

\author{
Marie-Dominique Marcant \\ Language Center, Université de Lausanne (Switzerland)
}

\begin{abstract}
The Language center at UNIL offers a twofold language training combining communication skills in a foreign language classroom with a professor as well as independent learning skills while working with advisors on independent learning skills in a multimedia center. Students from beginner level to A2 level have one hour and a half in the classroom with a professor and two times 45 minutes in the multimedia center where they can go at any time they want. Students from A2/B1 level to B2 level have one hour and a half in the classroom and 45 minutes in the multimedia center. This setting in which the students are working independently in the multimedia center is based on the theories of autonomy, and more specifically, on the concept of individual support for independent learning as defined by M.-J. Barbot (2006). M.-J. Barbot advocates for engaging the learner in his/her learning process by making him/her responsible for the path taken. In this perspective, the learning environment should provide tools and support to guide rather than teach the student in order for him/her to be able to pursue the learning process on a life-long basis. Based on this research, the goal of the action research project led at UNIL aims at improving the quality of the support an advisor can give to a student in his/her independent learning path. However, one of the major challenges for this support - as the advisors in the multimedia center are also the professors in the classroom but playing a different role - is a horizontal, nondirective, computer mediated communication between the learner and the advisor. Indeed, the advisor's role is not to teach, nor to follow the progress of a student but rather to take a student at a T-moment and manage to engage with him/her to understand what are his/her needs and expectations to then be able to suggest activities, strategies, etc. in order to help the student experiment new ways of learning and thus provide him/her with tools on a more and more independent path. Therefore, this computer mediated communication requires new skills and implies a paradigm change that has caused some hesitations from the professors in their new role of advisor. Initial hesitations by the team members included the fear to interact on a punctual basis with students they don't know, fear to interrupt and bother a student who is already engaged in an activity, unease to observe what students do on the computers as if they were spying, insecurity in the validity and quality of a feedback they could give to someone they don't "follow" in a classroom. Following these hesitations, the Language center led an action-based research to address the challenges in computer-mediated communication. To do so, the action research seeks to questions the system and the representations about the system by the users (advisors and students). The advisors were given a questionnaire about how they understood the concept of independent learning, about how they felt in their advisor role, what were the strengths and weaknesses of the system according to them, what would they change to improve it, etc. This questionnaire also had a list of ideas of what an advisor could do and the latter were asked whether they had already tried these actions or not, if they would like to try it or not and why. At the same time, the students received as well an online questionnaire about their practice, their use of the multimedia center, their feedback on the type of advice they received by the advisors, etc. In a second part, sessions in the multimedia center were observed by the researcher following an observation protocol sent to all the advisors beforehand with again the list of possible actions/interactions. After analyzing this data, the results were presented to the team and then some individual interviews were conducted in order to get an opportunity for each one to close the process and say what they felt during the study and if this had helped them to a certain extent or on the contrary provoked a supplementary stress. Finally, the team worked together on new tools to respond to the needs that came out of the study. This paper presents briefly the theoretical background and the data that has been used. It, then, discusses the results of this action-based research as well as the current and possible forthcoming tools and training that can be developed based on the findings.
\end{abstract}

Keywords: Language learning-teaching-advising, independent learning, computer-mediated learning. 


\section{Introduction}

The Language center at the University of Lausanne (UNIL) offers to the academic community (students, PhD, postdoc, faculty, staff) language training in 7 languages: English, German, French, Italian, Spanish, Russian and Chinese. Many of these courses consist of a twofold training targeting language learning and the acquisition of independent learning skills at the same time. The training takes place partly in the classroom with a teacher ( 90 minutes for all the levels) as well as in a multimedia center ( 90 minutes for beginner level to A2 level and 45 minutes for A2/B1 level and above). The multimedia center is a room with 30 computers and a database with a lot of materials of all kinds to practice the different skills at one's own pace and according to one's personal goals and needs. When at the multimedia center, students can choose what they would like to work on - go further on what has been learnt in the classroom, repeat what has been done or work on personal objectives for their work and/or personal life. They work independently but an advisor is present in the room to answer questions as well as to give support and tools to help the learner develop some learning strategies to improve his/her language learning skills. The advisor can see the 30 computers from his/her own computer and s/he can call students through headphones when needed and vice versa. The first week of the semester is dedicated to an introduction of the system for students to understand the concept, what they can find and what kind of support they can get. It is also important to note that the advisors are the language teachers but playing a different role following the theories of autonomous learning and particularly the work by Marie-José Barbot (2006, 2012).

\section{Theoretical framework}

In this setting, the teachers are asked a few hours per week to work as advisors at the multimedia center. Marie-José Barbot (2006) defines the "advising approach" as being "different from the traditional heterotraining educational system which takes as a starting point contents to radically move to the learner's side and thus toward complexity". She explains that one of the great difficulties is for the teacher to leave his/her usual and expected role to work toward a new role that will require a constant adaptation to the individual s/he is dealing with. According to M-J Barbot and M-J Gremmo (2012) a deconditioning phase is needed to go from a prescribing role to an advising role. The advising role provides support and tools for the development of language learning at the same time as the development of a learning culture. The advising role also provides with methodological support by suggesting activities and approaches to experiment and finally it also provides a psychological support by helping the student to objectify his/her difficulties and successes.

M-J Barbot and M-J Gremmo (2012) insist on the difficulty to change one's habits to reach this new role because there is a risk to use the computer-mediated approach to reproduce a transmissive and directive approach instead of developing autonomy and independent learning. They also underline the risk for the teacher to feel reduced to a secondary role without any clear goals and actions to do. This change of paradigm requires greater adaptability skills as it is necessary to be able to observe and listen to actually start from what the student needs - even if we meet the student for the first time - and not from a predefined content the teacher might have in mind.

\section{Challenges}

These challenges mentioned by M-J Barbot $(2006,2012)$ put the teacher in his/her new role of advisors often in an uncomfortable situation that demand to leave one's comfort zone and to take some distance with one's usual practice to start almost a new job.

The main challenges that team members at the Language center at UNIL felt really come from the change of paradigm: fear to interact with student one doesn't know and fear of not being able to give the appropriate support; fear of interrupting a student while s/he is working; discomfort with the idea of communicating though headphones while being in the same room; fear to be intrusive because of the possibility to view students'screen, etc.

Some team members often complain about this part of their work and question the efficiency of such a system and also wonder if students actually find any gain with this twofold learning environment. Some team members raised the issue of a potential paradox in claiming to train for independent learning but making it compulsory in the training while some students might prefer to work on other platform with other material. The latter is also a point of complaints from the team members that find the material too

\footnotetext{
${ }^{1}$ Our translation. Original text : «l'accompagnement se démarque du système classique éducatif d'hétéroformation qui part de contenus, pour se déplacer radicalement du côté de l'apprenant et de la complexité »
} 
old fashion and no longer adapted to today's learning culture. It became then necessary to question the system and try to put the light on what can be attributed to representation / projection and what actually are the strengths and drawbacks of such a system, to try, then, to tackle these hesitations.

\section{Methodology}

An action-based approach was chosen because the study aimed at improving the situation for the advisors as well as the students. This is why, the tools described below were also tools which allowed the users (advisors and students) to reflect on their practice and get new ideas though the study.

The coordinator of the project started with a literature review of the theories of autonomous learning. Then, a set of different methods were gathered to try to get a global view of the system: 1) the 29 teachers-advisors were asked to fill in a questionnaire regarding their understanding of the approach implemented at the Language center, the positive aspects and negative aspects of the system according to them and to mention what type of support/interaction they give / have while advising in the multimedia center. For this last part of the questionnaire a list of 26 items which were also ideas of what an advisor could do was given. 2) students were asked to fill in a questionnaire as well in order to gather information about whether they were going on a regular basis or not and why (not), what are the strengths and drawbacks according to them and how do they use the system. For this last part, like for the advisors, a list of items was given. 419 students (about 30\% of the total number of students who received the questionnaire) answered. The two questionnaires were then analyzed and put together in order to cross teachers' and students' speech to see where they meet and where they diverge. 3) a series of observations of sessions at the multimedia center were conducted. Each advisor was observed 3 times: once at the beginning of the semester when the system is presented to the students, once in the middle when students get some weekly ideas of what to do as they $\log$ on, and once toward the end when students no longer receive the suggested activities. These observations followed a protocol that was presented to the team beforehand to be able to get their comments and feedback. These observations were analyzed and then crossed with what advisors said they did or not in the questionnaire. 4) Individual interviews were conducted with each advisor in order to get a final feedback on how they felt throughout the study, on what they would like to say about the approach, etc. 5) Finally, the results were presented to the team and the team collaborated in order to produce new tools to help the advisors' work.

\section{Results}

\subsection{Understanding the concept}

The answers from the teachers-advisors to the question on how they would define the concept of advising/counseling in the context of an independent learning environment such as the multimedia room at the Language center at UNIL show a general understanding and agreement on the objectives even though a close look at the wording show that the definition are all going toward a centration on the learner but the actions that are mentioned can be divided in three categories: diagnostic actions ("observe", "listen", etc.), support actions ("support", "help", "advise", etc.) and structuring actions ("follow", "oversee", "supervise", etc.). The two first categories are fully part of the work of an advisor but the third category matches more what is expected of a teaching situation with a program to follow knowing the learners and being able to supervise their progression which is not possible in the current setting as in each session new students can come and the advisors are expected to be able to make a diagnosis and provide support for this specific session.

\subsection{Perception of the dispositive}

The figures below show the way some aspects of the learning environment are perceived by the different users: advisors and learners based on answers to the questionnaires about the strengths and weaknesses of the system.

The first figure shows that globally students and advisors agree on the positive aspect of having the presence and support of an advisor and with the possibility to work on personal learning objectives. Teachers-advisors have put more values on the amount of available material while students don't mention it as much in the strengths of the dispositive. 
Figure 1. Strengths of the dispositive according to the users.

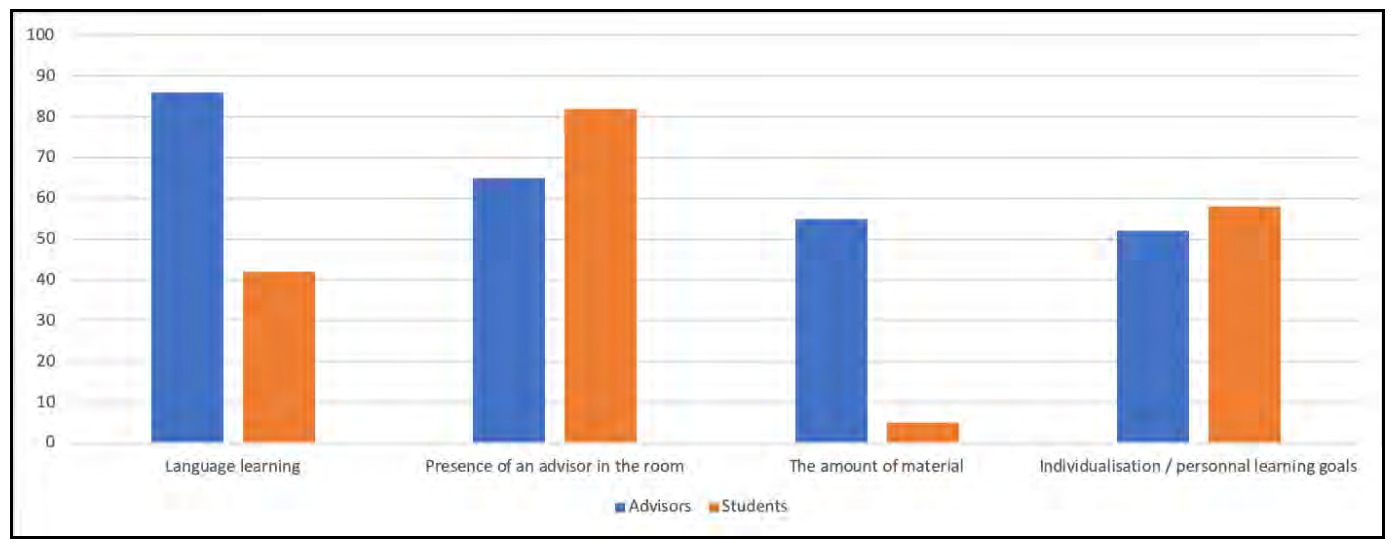

The second figure show also a very similar perception of the drawbacks for teachers-advisors and students except for the oldness of the material for which students don't really comment on while teachers-advisors point at it as a main drawback of the system.

Figure 2. Drawbacks of the dispositive according to the users.

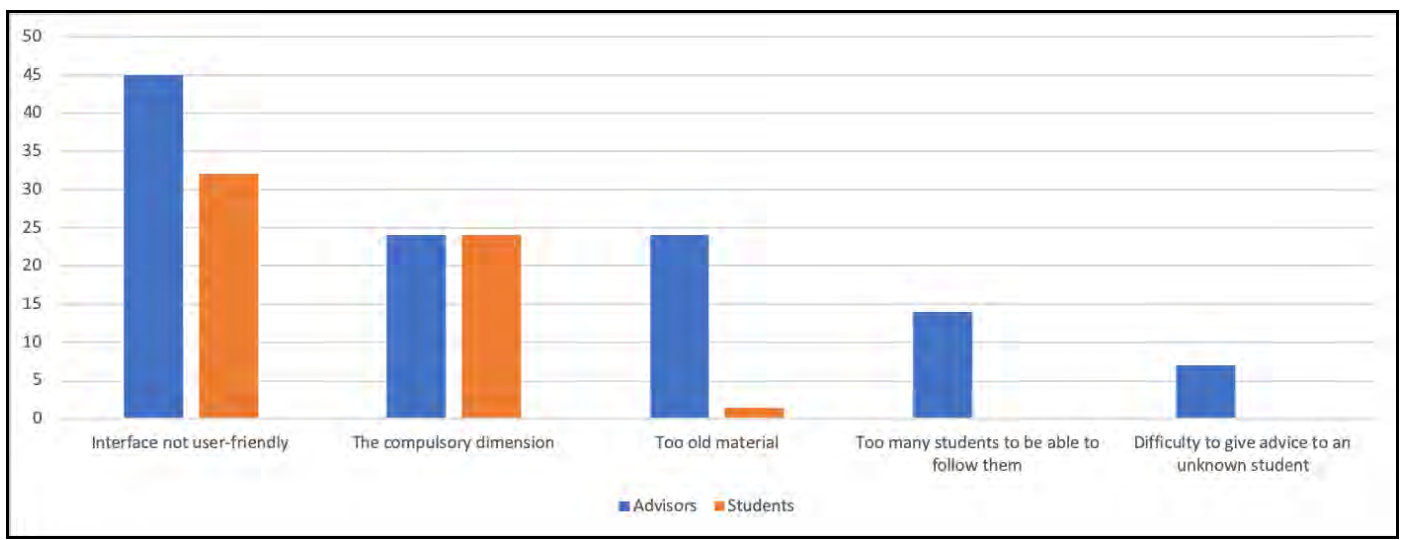

Both students and teachers-advisors agree on the fact that the system is not very intuitive or user-friendly. Students don't always easily find activities that match their expectations and level. This shows a problem that can come from the available material and/or the search engine of the data base.

The fact that the sessions are compulsory are equally mentioned by students and team members. There is a demand for the system to be online for more flexibility. However, the current system allows students to be sure to be able to receive feedback as they are working which is seen as a strength as seen in the first figure. In the second figure above, we also see the challenges of the advisors to work with students they don't know and the feeling of not being able to do a quality job as there is no possibility for a real follow-up. However, the third figure below somehow contradicts this impression of the teachers-advisors. This figure below shows the answers of the students to the question on why they were attending (or not) the independent learning sessions. It is clear that, globally, students are satisfied by the support they receive from the advisors and the fear of the teachers-advisors of being intrusive, of interrupting the work is only felt by very few students.

Figure 3. Reasons given by students on why (not) they attend regularly the independent learning sessions.

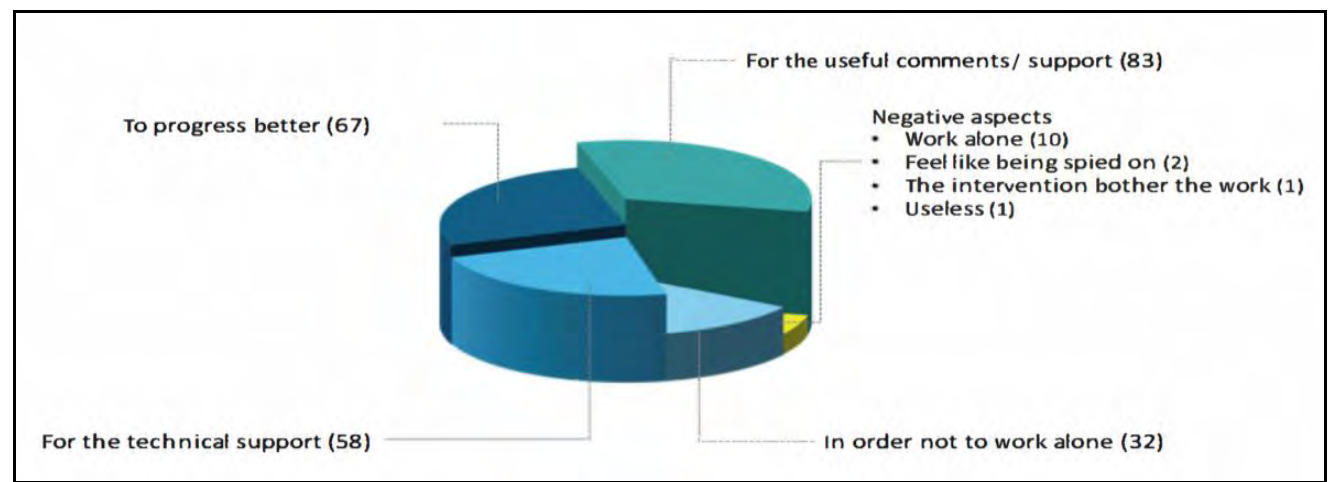


These results show some representations and/or projections from the advisors' point of view that is not perceived strongly in the answers of the students.

When looking at the teachers-advisors answers to the question about what type of support they were giving (choosing from a list) and crossing these answers with the observations, it is also interesting to mention that we find a total adequation between what advisors say they do and what is observed when dealing with short targeted interventions (answering a question, suggesting an exercise, explaining how to find material in the room, etc.). When it comes to actions that are more related to the development of independent learning we see a gap between what advisors say they do and the answers of the students, who say they did not receive this type of support and the observations that didn't show this type of intervention. These actions - such as referring to the plurilingual profile of the learner to help comparing and understanding the target language, helping the student to set goals, helping the student to experiment new strategies, etc. - are very meaningful but also very demanding as it requires time to be able to understand where the student is in his/her learning process, what are his/her needs, what s/he has already tried, etc. Teachers-advisors feel they have to give the same amount of support to everyone in each session as if it was a class. These actions requiring more time, are thus much less used. This shows a challenge in the change of paradigm from teaching to advising: they know what is expected from them in their advisor role but it is still challenging to put it all in practice as some habits are deeply anchored in the practice. In fact, in the individual interviews conducted at the end of the project, advisors showed some insecurity toward the strategies they are asked to use to help the students and it appeared that the interactions were maybe too often put into place through "weak questioning" such as "how are you?", "Is everything going well?" in order to gain time but which does not necessarily engage the student toward questioning his/her work. So, some students might just say they are fine ending this way the interaction. This might be one aspect that create this impression of not being able to help in a meaningful way the students.

\section{Impact}

This action-research has already had several impacts: the ideas given in the questionnaires were felt as an actual help with more support and ideas. Some of the team members said that the fact of being observed by another colleague pushed them to try to interact more with students and felt more useful as the interaction was often more fruitful than they thought.

The other impact of the action-research is that the study allowed us to get feedback and ideas to develop tools to tackle the existing insecurities. The team shared all the strategies that they had in mind and these were organized by skills/objectives (listen, read, setting goals, etc.) in an Excel sheet. This repertoire of strategies is now available to all advisors who would need more ideas. The team also worked on how to start an interaction with the students to avoid weak questions that don't engage the learner in the learning process. The team came up with a timeline of different stage of the student's activity and ideas of questions to start the communication.

\section{Perspective for the future}

This study has shown that this twofold learning environment is appreciated by the users however the idea of a computer room can seem a bit out of date and rigid for students with very busy schedule. At a time when everything can be found on the Internet it might seem old-fashion to impose on students to come study in a computer lab. However, this type of setting does have benefits. We see a need to dematerialize space, to increase flexibility and easy access but in cities the co-working spaces are booming, libraries are still used by students as working spaces. This reveals that despite the flexibility the technology can offer, many people still need to be able to create time and space dedicated for one's activity. This prevents being interrupted, or find other things to avoid doing an activity. Dedicating time and space for an activity give incentive to be more regular and guarantee better quality in the learning process. This is why, for the future, it is important to question how can we offer the incentive while also offering more flexibility and a setting more in adequation to today's use of technologies.

\section{References}

Barbot, M.-J. (2006). Rôle de l'enseignant-formateur : l'accompagnement en question. Mélanges Crapel, $n^{\circ} 28$, pp. 29-46.

Barbot, M.-J., Gremmo, M.-J. (2012). Autonomie et langues étrangères: Réaffirmer l'héritage pour répondre aux nouveaux rendez-vous. Synergies France, ${ }^{\circ}{ }^{9}$, pp.15-27. 